\title{
Se passer de la monnaie : la banque d'échange de Victor-Corentin Bonnard (1849-1862)
}

\section{Nicolas Stoskopf}

\section{(2) OpenEdition}

\section{Journals}

Édition électronique

URL : http://journals.openedition.org/ress/227

DOI : $10.4000 /$ ress.227

ISSN : $1663-4446$

Éditeur

Librairie Droz

Édition imprimée

Date de publication : 1 juillet 2007

Pagination : 167-175

ISBN : 978-2-600-01155-6

ISSN : 0048-8046

\section{Référence électronique}

Nicolas Stoskopf, « Se passer de la monnaie : la banque d'échange de Victor-Corentin Bonnard (1849-1862) », Revue européenne des sciences sociales [En ligne], XLV-137 | 2007, mis en ligne le 01 juillet 2010, consulté le 04 mai 2019. URL : http://journals.openedition.org/ress/227 ; DOI : 10.4000/ ress. 227 
Nicolas STOSKOPF

\section{SE PASSER DE LA MONNAIE : LA BANQUE D'ÉCHANGE DE VICTOR-CORENTIN BONNARD (1849-1862)}

La monnaie, personnage historique? Oui, mais dans le rôle de l'homme invisible ! C'est le scénario proposé par les promoteurs des banques d'échange qui se sont multipliées au milieu du XIX ${ }^{\mathrm{e}}$ siècle dans le contexte de la crise financière de 1848 .

L'idée de la banque d'échange n'était pourtant pas nouvelle : elle était pour ainsi dire née avec le socialisme comme un moyen d'affranchir le producteur du capitaliste et de donner au travail une juste et exacte rémunération en court-circuitant le capital et en se passant de monnaie. Robert Owen fonda ainsi à Londres en 1822 une banque d'échange où la monnaie était remplacée par des bons d'heures de travail. En France, un certain Fulcrand Mazel tenta également l'expérience en 1829. Puis ce fut au tour de Proudhon et de sa Banque du Peuple : fondée le 11 janvier 1849, elle cessa ses opérations au bout de deux mois sans avoir véritablement fonctionné ${ }^{1}$. De nombreuses autres tentatives se soldèrent par des échecs ${ }^{2}$.

La banque d'échange de Victor-Corentin Bonnard fait exception : ouverte à Marseille le 10 janvier 1849, c'est-à-dire un jour avant celle de Proudhon, elle connut un succès éclatant qui incita Bonnard à fonder un établissement à Paris en 1853. Pour nous qui connaissons la fin de l'histoire et le triomphe sans partage de l'échange monétaire, cette aventure ne laisse pas d'étonner : avant de se terminer en impasse, la voie ouverte par Bonnard a pu paraître prometteuse ; il a su convaincre ses partenaires et ses clients, tous des professionnels, de l'efficacité de son système ; ceux-ci ont pu croire que la monnaie serait mise au rang des accessoires et que l'avenir serait différent. C'est cette illusion collective qui mérite d'être étudiée dans la mesure où elle renseigne sur la perte de confiance subie par notre personnage historique au milieu du XIX ${ }^{\mathrm{e}}$ siècle, une sorte de traversée du désert qui est à vrai dire le lot commun de tous les personnages historiques !

On dispose d'une documentation importante et variée, exceptionnelle pour un établissement de ce type : les Souvenirs de famille, publiés en 1934 par Joseph $\mathrm{Naud}^{3}$, petit-fils de Bonnard, constituent une base narrative précieuse, qui doit être complétée par les papiers de l'entreprise (actes de société, procès-verbaux d'assemblée générale) et par des publications commerciales (brochures, catalogues, factums). L'une de ces brochures présente un intérêt particulier : intitulée Comptoir

1 Sur ces tentatives, voir Vrégille (1933).

2 Selon Bonnard, 33 banques d'échange auraient fonctionné à Marseille en 1849, 200 dans toute la France. D'après le compte rendu à l'assemblée générale des actionnaires du 26/01/1856, rapportée par Lechevalier Saint-André (1858).

3 Naud (1934). 
central, séance du 13 juin 1853 chez Lemardelay ${ }^{4}$, c'est le compte rendu sténographié de la rencontre entre Bonnard et 180 personnes, la plupart appartenant au syndicat des épiciers en détail, les autres étant « quelques journalistes, de hauts personnages et des négociants appartenant à divers autres genres de commerce $»^{5}$. Bonnard y a la parole, mais les arguments s'échangent et l'opinion publique s'exprime.

\section{L'AVENTURE DE BONNARD}

Victor-Corentin Bonnard, qui n'appartenait pas à la mouvance socialiste, était un pur produit du milieu commercial. Ses origines sont modestes : né à Songeons dans l'Oise le 26 janvier 1804, il était le fils d'un percepteur, qui avait été secrétaire de Bernadotte pendant les campagnes de l'Empire. Sa mère étant morte peu après sa naissance, il fut élevé par sa tante, mariée à un aubergiste. On ignore tout de sa jeunesse ; on le retrouve vers 1825 à Smyrne, où il établit des relations commerciales avec Marseille. Il s'installa ensuite à Marseille, s'y maria en 1831 et vécut assez chichement pendant quelque temps. Ses affaires finirent néanmoins par lui procurer une certaine aisance. A partir de 1847, il envoya sa famille à Paris durant l'année scolaire afin que ses enfants puissent faire de bonnes études : de fait, son fils cadet entra à l'Ecole polytechnique.

C'est donc au contact du commerce marseillais, et après une bonne vingtaine d'années d'expérience, que germa l'idée de la banque d'échange : l'objectif était d'apporter davantage de souplesse au troc habituellement pratiqué par les commerçants marseillais entre eux, tout en évitant de stériliser un stock; au lieu de centraliser dans un bazar des marchandises en quantité et en choix nécessairement limités, la banque d'échange se contentait de détenir en portefeuille des bons signés par les commerçants et payables en marchandises. L'acheteur se procurait immédiatement la marchandise ou le service de son choix en payant une commission en espèce et en remettant un bon libellé en francs, mais payable en marchandises ou en services qu'il s'engageait à honorer sur simple présentation. La monnaie n'était donc pas totalement évacuée, puisqu'elle servait toujours à mesurer les prix et... à rémunérer la banque. Celle-ci détenait en portefeuille des bons d'échange (ou « billets de crédit») qui étaient autant de créances sur le travail ou sur les marchandises des artisans et des commerçants de la place ${ }^{6}$. Ses clients se procuraient ces bons en souscrivant à leur tour des bons sur leur propre travail.

Bonnard répétait à l'envi que sa première affaire avait été, le 10 août 1838 , l'échange d'une paire de botte contre un parapluie ${ }^{7}$. Cela devint la date mythique de la fondation de l'entreprise. En réalité, il attendit encore plus de dix ans pour donner à celle-ci une dimension significative. La crise de 1848 lui offrit des conditions favorables. Dès août, il intervint dans la presse marseillaise pour critiquer les

4 Lemardelay (1853) Nous remercions vivement M. Georges Mouradian d'avoir bien voulu nous faciliter la consultation de ce document.

5 Lemardelay (1853), p. 4.

6 Le journaliste Eugène Forcade assimile le billet de crédit à « un warrant personnel qui constitue le souscripteur du billet débiteur à présentation de la somme en marchandises et au cours du jour exprimée sur le billet; seulement, la marchandise n'est point déposée dans un entrepôt public : c'est le souscripteur du billet qui en reste détenteur. » Forcade (1858).

7 Lemardelay (1853), p. 23. 
officines pratiquant l'échange et lança des appels à la souscription d'un capital en faisant valoir les avantages de sa formule. Il ne fut guère entendu : sur 20000 actions émises à 25 francs seulement, il n'en écoula que 313 qui lui apportèrent 7825 francs. Il mit dans l'affaire toutes ses économies, soit 15000 francs, et créa le 10 janvier 1849 la Banque d'échange de Marseille sous forme d'une société en commandite par actions. Le succès fut au rendez-vous. En 1852, le capital prévu initialement était enfin souscrit et une seconde tranche équivalente fut introduite en bourse. Le 5 janvier 1853, Bonnard put annoncer avec fierté aux actionnaires que les titres, payés 25 francs, avaient rapporté 84 francs, étaient cotés 82 francs, et qu'il leur avait donc rendu six fois et demi ce qu'ils lui avaient confié. Une assemblée générale extraordinaire du 29 mars 1853 l'autorisa à fonder le Comptoir central de crédit à Paris et à créer des succursales en province.

Très peu connu à Paris, Bonnard eut à nouveau de grandes difficultés pour réunir le capital nécessaire : la société démarra le 24 mai 1853 avec 625000 francs au lieu des deux millions attendus. Cette sous-capitalisation était le point faible traditionnel des sociétés financières en commandite par action : les épargnants répugnaient à confier leur argent à un inconnu et réclamaient des dividendes élevés qui empêchaient l'autofinancement. C'est pourquoi Bonnard fit notamment entrer dans son conseil d'administration des notables respectables, quatre sénateurs, dont le marquis de Lagrange, un député, l'orfèvre Charles Odiot et le raffineur de sucre Arnaud Jeanti. Le lendemain de la réunion chez Lemardelay, soit le 14 juin, le Comptoir central commença ses opérations au 51, rue de la Chaussée d'Antin.

Trois ans plus tard, Victor-Corentin Bonnard avait fait fortune : en 1856, le Comptoir acheta, pour près de deux millions de francs, l'hôtel Talhouët-Roy, 66, rue de la Chaussée d'Antin, où Bonnard emménagea avec sa famille. Il dépensa 200000 francs pour le mobilier et organisa de brillantes réceptions. L'année suivante, le Comptoir fit l'acquisition du château des princes de Conti à Issy. Les Bonnard se montraient dans les chasses à courre. C'était l'apogée.

\section{LES RAISONS DU SUCCÈS}

Comment expliquer ce succès ? Les raisons doivent en être cherchées dans le contexte des difficultés de paiement et de crédit au milieu du XIX ${ }^{\mathrm{e}}$ siècle et dans les avantages propres du système Bonnard.

Le premier point peut être illustré par le récit d'Edmond Werdet, un ancien libraire contraint de cesser ses activités en 1839, décrivant le quotidien du commerçant, aux prises avec les difficultés de trésorerie qui le conduisent chez l'escompteur, "un monstre spongieux, une sangsue, un ogre, un vampire... les effets de commerce [...] étaient apportés, deux fois par mois, quelquefois davantage, dans l' antre du minotaure, pour y être convertis en espèces sonnantes, moyennant un escompte régulier de $3 / 4 \%$ mensuel, soit $9 \%$ par an, escorté de prime, d'agio, de commission, de change de place, de courtage, de sorte que l'obligeant ami, qui s' informait constamment, avec la sollicitude d'un père, de la santé de son client, de son écorché tout vif et des siens, prélevait sans la moindre vergogne le plus pur des bénéfices de la boutique, soit de 10 à $12 \% »^{8}$.

\footnotetext{
8 Werdet (1860), pp. 325-326.
} 
La crise financière de 1848 , en provoquant une déflation brutale, ne fit que rendre plus aiguë la pénurie de numéraire et la hantise de la cessation de paiement, c'est-à-dire de la faillite. Elle détruisit le système de crédit laborieusement mis en place au cours de la Monarchie de Juillet et eut au moins le mérite de susciter des solutions inédites : comptoirs d'escompte à Paris et en province en 1848, caisses d'escompte d'Alfred Prost en avril 1852, projet Pereire de caisse centrale des sociétés de crédit mutuel au service de l'artisanat et du commerce ${ }^{9}$, banques d'échange, dont celle de Bonnard.

Celui-ci réalisait au profit de ses clients une sorte de petit miracle que l'on croirait tout droit sorti des propos d'un bonimenteur ; il leur offrait deux avantages, un capital, sous forme de marchandises à prendre chez un fournisseur, et une clientèle, puisqu'il se chargeait d'écouler les bons souscrits par le commerçant. En contrepartie, le bénéficiaire «gardait ses écus »" à l'exception du paiement de la commission. L'essentiel tenait donc dans ce crédit sans intérêt (et sans argent) qui répondait à l'un des principes de bases énoncé par Bonnard, «faire que dans toute transaction, chacun puisse être payé d'avance au lieu de n'être payé qu'après l' achat, suivant l' usage établi ${ }^{11}$. Autrement dit, le commerçant ou l'artisan étaient payés avant même d'avoir vendu !

Du même coup, ce crédit présentait la particularité de courir en sens inverse du crédit commercial ordinaire : il ne naissait pas d'affaires faites et réalisées, mais d'affaires à venir, et était accordé au producteur par le consommateur, dont la banque tenait la place ${ }^{12}$. Il revenait à celle-ci de boucler l'opération en écoulant les bons souscrits par le débiteur. Ce dernier était en principe délié de tout souci, sauf de tenir la marchandise prête et de répondre à toute présentation d'un bon, il n'était pas sous la dépendance de la banque et n'était pas son obligé : « Si j’avais l'honneur de faire un crédit de 10000 francs à quelqu'un d'entre vous, déclara Bonnard aux épiciers, et que je vinsse lui demander pour 5 francs de marchandises, il pourrait [...] m' envoyer promener $\gg{ }^{13}$. De son côté, la banque n'engageait pas son capital dans ces opérations de crédit à l'exception de ce qui avait été nécessaire pour l'amorçage de la pompe ou pour se procurer des marchandises impossibles à obtenir par la voie de l'échange. Elle n'endossait pas le billet de crédit, le transmettait à ses clients sans engager sa responsabilité, ce qui libérait son capital pour d'autres usages : ainsi, dans le bilan de 1858 , le poste « billets de crédit » ne comptait que pour 4,3 millions de francs sur un actif de 22,7 millions de francs $^{14}$.

Bonnard voulut donner en outre à son entreprise une dimension morale et philanthropique. A la question de savoir s'il n'accorderait pas sa préférence à ceux

9 Rejeté par le Conseil d'Etat en mai 1853.

${ }^{10}$ Lemardelay (1853), p. 9.

${ }^{11}$ Lemardelay (1853), p. 7. En 1849, Bonnard écrit au moment du lancement de la Banque d'échange de Marseille : «La société ne demande jamais aux échangistes de livrer leurs marchandises à découvert ; l'échangiste ne souscrit un bon qu'autant qu'il a reçu immédiatement l'équivalent en marchandises ou en travaux de son choix » Banque (1849).

${ }^{12}$ Courcelle-Seneuil (1857), pp. 389-390.

13 Lemardelay (1853), p. 12.

${ }^{14}$ AG des actionnaires, 31/01/1857, pp. 11-12 (CAMT 65AQ A 390). 
qui lui paieraient la plus forte commission, il répondit : "Nous n'avons de préférence que pour celui qui souffre $»^{15}$. Au-delà de la déclaration d'intention, il est clair que la raison d'être du système était d'apporter une aide à des commerçants dans la gêne. Il fit également inscrire dans les statuts une clause prévoyant la distribution de $10 \%$ des bénéfices à des œuvres d'utilité publique. Un bureau se chargeait d'instruire les demandes de secours. Il intervint pour aider des familles pauvres à se dégager du Mont-de-Piété, envoya des aides aux inondés du Midi ou aux ambulances de Crimée et donna 150000 francs à la ville de Marseille pour la construction d'une école.

Il ne faut pas voir néanmoins dans ces dispositions les signes d'une attirance pour le socialisme. Bonnard ne remettait pas en cause les règles de l'échange monétaire et encore moins du capitalisme ; il prétendait simplement en corriger certains défauts, faciliter le commerce en mettant en relation vendeur et acheteur, et accroître la demande. Ainsi, il attribuait à son système des vertus anti-crise : «En temps de crise, l'argent ne circule pas, mais la consommation existe. Si l'on peut alimenter sa consommation sans donner d'argent, grâce à notre concours, il faut reconnaître que le Comptoir est bien une des institutions les plus utiles pour la société, surtout dans les jours difficiles.» Il attendait de la mise en œuvre de ses principes « une augmentation considérable de la consommation». Ses clients ne devaient pas enregistrer une diminution de leur vente contre argent et exercer une concurrence déloyale vis-à-vis des autres marchands, mais au contraire réaliser des ventes qu'ils n'auraient pas faites autrement ${ }^{16}$.

De fait, Bonnard entendait moins créer un circuit concurrent que complémentaire. Quant au bon d'échange (ou billet de crédit), il n'est pas assimilable à une monnaie parallèle, entrant en concurrence avec la monnaie officielle. Nominatif et portant sur une marchandise précise, il n'était pas endossable et sa circulation se limitait à une boucle simple et stéréotypée : émetteur-banque-acheteur-émetteur. C'était précisément un de ses avantages par rapports aux bons émis par d'autres banques d'échange ${ }^{17}$ : chez Proudhon, ces bons circulaient entre les adhérents de la Banque du Peuple ${ }^{18}$ et devaient être acceptés pour tous les paiements. Entrant en concurrence avec la monnaie, le bon était inévitablement déprécié si bien que le vendeur faisait la loi à l'acheteur, relevant ses prix pour la circonstance ou livrant une marchandise de moindre qualité. En principe, ce type de mésaventure ne devait pas se produire pour les clients de Bonnard : l'acheteur traitait librement, dans les conditions ordinaires du marché, sans être obligé de se déclarer comme client de la banque d'échange, et ne remettait le bon qu'au moment de payer; le vendeur ne pouvait renier sa propre signature, d'autant que le bon portait la mention suivante : «Je m'oblige à payer en espèces, si je n'étais pas en mesure de remplir mon engagement $\gg$.

Bonnard apportait incontestablement des solutions aux problèmes rencontrés par le commerce. Son idée fut favorablement accueillie par les économistes.

15 Lemardelay (1853), p. 15.

${ }^{16}$ Lemardelay (1853), pp. 40, 48 et 30.

17 Dites «banques monétaires d'échange » par Courcelle-Seneuil (1857), p. 380.

18 A la différence de la Banque du peuple, Bonnard ne restreignait pas l'échange aux actionnairesadhérents. 
Courcelle-Seneuil par exemple n'y voyait pas une «panacée sociale », mais un "procédé, une simplification apportée dans le mécanisme des échanges, quelque chose comme le retranchement d' un ou de deux engrenages dans une machine », c'est-à-dire une véritable « innovation apportant comme tous les procédés, qui économisent un emploi de temps et de travail, des richesses nouvelles à la société $\gg{ }^{19}$.

\section{LIMITES ET ÉCHEC}

En réalité, le système atteint assez rapidement ses limites et Bonnard fut mis en difficulté à partir de 1857. D'abord, il se heurta à l'inertie des commerçants peu enclins à changer leurs habitudes de travail. Bonnard avoua lui-même devant les épiciers parisiens qu'il avait eu beaucoup de répugnances à vaincre à Marseille ${ }^{20}$. A Paris, les questions posées par ces épiciers sont révélatrices de leurs inquiétudes : les uns craignaient de voir supprimer les intermédiaires, d'autres d'avoir affaire à un intermédiaire de plus. La question de la commission suscita un feu nourri de questions et d'objections : Bonnard n'allait-il pas fixer la commission à la tête du client, favoriser les commerçants qui accepteraient le taux le plus élevé, entraîner la concentration, voire le monopole ? Comme le système était fondé sur des transactions en chaîne, on soupçonnait Bonnard de vouloir accaparer les bénéfices du commerce. Certains, s'exagérant les chances de succès de Bonnard, le voyaient déjà exercer un monopole et prélever une commission de $3 \%$ sur l'ensemble des échanges ${ }^{21}$ ! La plupart de ces craintes étaient vaines. Jules Lechevalier Saint-André ne nous surprend pas outre mesure en nous apprenant en 1856 que « les billets de crédit n' ont pas pénétré dans les maisons établies de longue date » et que «les difficultés de Bonnard tiennent à la résistance du milieu dans lequel il opère $»^{22}$.

Il faut croire que, pour beaucoup, le jeu n'en valait pas la chandelle. Le système n'était pas exempt de défauts, ceux-là même que l'usage de la monnaie permet d'éviter : le bon d'échange rendait plus difficile, sans l'empêcher complètement, la liberté de choix du fournisseur et la satisfaction des convenances personnelles. Il n'était pas facilement divisible et les solutions suggérées par Bonnard lui-même, souscrire des bons de toutes les coupures, depuis un franc jusqu'à mille francs, rendre la monnaie par un nouveau bon du montant de la marchandise restant à livrer ${ }^{23}$, n'étaient que des pis-aller malcommodes, insupportables même en dehors des périodes bien spécifiques de gêne monétaire.

Enfin et surtout, la charge incombant à la banque devint rapidement excessive, d'autant qu'elle devait assumer deux tâches contradictoires, trouver une clientèle pour tous les professionnels qui alimentaient son portefeuille et réguler l'émission de nouveaux bons en fonction des possibilités du marché. " C'est à moi de prendre des précautions », disait Bonnard, «[...] je sais que je dois avoir à

\footnotetext{
19 Courcelle-Seneuil (1857), p. 390.

${ }^{20}$ Lemardelay (1853), p. 42.

${ }^{21}$ Lemardelay (1853), pp. 13, 15, 16, 33, 47, etc.

22 Lechevalier Saint-André (1858).

${ }^{23}$ Lemardelay (1853), pp. 31-32.
} 
Marseille que tant de cuivre, tant de métaux, parce que je n'ai qu' un capital de tant, et qu'il n'y a dans cette ville qu' une consommation de tant $»^{24}$. Le problème est que ces précautions constituaient autant de freins à la recherche des débouchés : la banque devait sélectionner sévèrement chacun de ses clients-acheteurs en fonction des marchandises ou des services qu'il offrait lui-même et pratiquer en permanence une sorte d'autocensure !

Concrètement, cela supposait la mise en place d'une administration très lourde : chaque matin, à sept heures, se tenait l'Ordre, rassemblant tout le personnel autour du gérant, où étaient discutées les propositions d'affaires ${ }^{25}$. Après consultation des fiches établies par la division des renseignements, on repoussait les $3 / 4$ des affaires proposées. Dans la journée, une armée de courtiers, rémunérés à la commission, parcouraient la ville pour trouver des nouveaux clients. A partir de 1857, un catalogue, contenant plus de 1500 adresses de clients de la banque classés par profession, fut diffusée ${ }^{26}$.

Mais malgré cette organisation, il était inévitable que le Comptoir, conçu pour les échanges entre professionnels, et donc pour des biens intermédiaires, finisse par être encombré de billets pour des produits finis à commercialisation lente et fractionnée, voire pour des marchandises invendables. C'était la sanction inévitable du principal avantage du système : celui-ci n'était pas fondé, comme le troc, sur la réciprocité des échanges, mais maintenait au contraire leur multilatéralité. Comme dans la loi de Gresham, la « bonne marchandise » trouvait preneur sans problème, alors que la «mauvaise » stagnait dans le portefeuille du Comptoir. La durée jouait donc contre le système qui subissait une sorte de freinage systémique, le condamnant à terme. Son espérance de vie, dans le cadre du système technique du XIX ${ }^{\mathrm{e}}$ siècle, était nécessairement limitée. Après avoir atteint 200000 francs de chiffre d'affaires et 4000 francs de commissions par jour, celles-ci retombèrent à 10000 francs par mois en $1857 \ldots$

Face à cette situation, Bonnard chercha à diversifier ses activités : il se lança dans la promotion immobilière, avec l'idée d'y appliquer les mêmes principes en vendant les parcelles en partie contre du numéraire, en partie contre des marchandises, des matériaux de construction par exemple, ou des travaux ${ }^{27}$. Il immobilisa la majeure partie de son capital entre 1855 et 1857 dans d'importantes acquisitions foncières : 255 hectares dans les communes de Boulogne, d'Issy et de Maisons-Laffitte qu'il paya plus de 19 millions de francs ${ }^{28}$. En mai 1856, il se rendit, sans succès, à Turin pour négocier la concession de 60000 hectares de terrains à coloniser en Sardaigne.

L'exercice 1857-1858 fut médiocre et le cours de l'action se retourna en 1858, tombant de 155 à 55 francs. Un actionnaire et client l'attaqua devant le Tribunal

${ }^{24}$ Lemardelay (1853), pp. 25 et 39 . Les marchandises étaient classées en deux catégories, $\mathrm{n}^{\circ} 1$ pour les produits finis, $\mathrm{n}^{\circ} 2$ pour les matières premières.

${ }^{25}$ La plupart des employés (une quarantaine en 1856 à Paris) arrivaient avant 6 heures et terminaient leur journée vers $19 \mathrm{~h} 30$. D'après Comptoir central (1856).

${ }^{26}$ Comptoir (1857).

27 Selon la répartition suivante : $1 / 10^{\mathrm{e}}$ au comptant en numéraire ; $3 / 10^{\mathrm{e}}$ dans 3 ans à $4 \%$ d'intérêt ; 6/10 en marchandises. Comptoir central (1856).

${ }^{28}$ Sur le sort de ces terrains, $c f$. Becchia (1978). 
de commerce et publia un libelle diffamatoire. A deux reprises, une cabale éclata au sein de son conseil de surveillance et parmi le personnel qui lui reprochait sa mégalomanie. Bonnard réussit à renverser la situation. Mais chaque assemblée générale devint une épreuve où il était attaqué de toutes parts. En 1862, il dut liquider sa fortune personnelle, plus de deux millions, pour éponger les pertes. Finalement le vicomte Lemercier, président de la société, le convainquit de se retirer en octobre 1862 en échange d'une succession garantie pour son gendre. Ces accords furent entérinés par l'assemblée générale du 19 mars 1863 qui nomma Edouard Naud à la gérance : V. C. Bonnard \& Cie devint alors E. Naud \& Cie. A la fin de 1865 , Bonnard ne possédait plus aucune action de la société qu'il avait fondée. Accablé de surcroît par la mort successive de ses enfants, aveugle et ruiné, Bonnard mourut le 25 janvier 1869 dans l'immeuble du 51, rue de la Chaussée d'Antin où il s'était réfugié.

\section{CONCLUSION}

Avec le recul du temps, l'expérience Bonnard apparaît comme une tentative sans lendemain, une curiosité datée, correspondant à la pénurie de crédit du milieu du XIX ${ }^{\mathrm{e}}$ siècle. Elle rendit momentanément des services en économisant l'emploi de la monnaie, en soulageant les trésoreries et en dispensant les usagers d'un capital plus important. Dès que l'argent devint plus abondant, elle perdit de son utilité d'autant que son fonctionnement finit par se gripper.

Et pourtant, le Comptoir central de crédit à Paris survécut ! De façon symptomatique, il fit à nouveau parler de lui en temps de crise, par un article de la revue Banque paru en 1933. L'économiste Gonzague de Vrégille, après avoir rendu hommage à Bonnard et souligné ce «quelque chose de génial » mis par lui dans les mécanismes de sa banque, indiqua les correctifs apportés au système depuis sa fondation : remboursement du billet de crédit en espèces au bout de trois ans s'il n'avait pas trouvé preneur ; paiements pour moitié seulement en billets de crédit, le reste en espèces ; circulation limitée au secteur du bâtiment et entre professionnels du BTP. Vrégille, optant pour la généralisation du billet de crédit $^{29}$, y voyait un moyen de lutte contre la crise et une solution au prétendu problème du moment, la surproduction : «Le banquier ne serait pas un simple vendeur de crédit; il serait tout naturellement amené à faire [...] de l'économie dirigée. Par là, une surproduction inconsidérée serait rendue particulièrement difficile, sinon impossible $»^{30}$. Les produits pourraient s'échanger contre des produits.

\section{SOURCES MANUSCRITES}

Actes de société, Maître Dupont, Archives nationales MC CXVIII-1043.

29 Sans toutefois se passer tout à fait de monnaie, « moyen de paiement tellement rapide et commode que son utilité lui a conféré des droits à jamais imprescriptibles », d'après Vrégille, (1933), p. 94.

30 Vrégille, (1933), p. 94. 


\section{SOUCES IMPRIMÉES}

Assemblées générales des actionnaires, coupures de presse et diverses brochures dont Comptoir central, séance du 13 juin 1853 chez Lemardelay, Paris, 1853, 52 p., Centre des archives du monde du travail, 65AQ A 390.

Banque d'échange de Marseille C. Bonnard \& Cie, Marseille, 1849, 24 p. (BNF 8.Vp.10108).

Comptoir central V.C. Bonnard \& Cie, Paris, 1856, 23 p. (BNF 8.V.11380).

Comptoir central V.C. Bonnard \& Cie, articles disponibles, 27 janvier 1857, Paris, 1857, 63 p. (BNF 4.V.4201).

Courcelle-Seneuil, Jean-Gustave, Traité théorique et pratique des opérations de banque, Paris, 1857.

Forcade, Eugène, Semaine financière, 13/02/1858.

Lechevalier Saint-André, Jules, Mémoire sur les institutions de crédit et en particulier sur le Comptoir central de crédit..., Paris, 1858, XIII + 64 p.

Werdet, Edmond, De la librairie française, son passé, son présent, son avenir, Paris, 1860.

\section{BIBLIOGRAPHIE}

Becchia, Alain, « Les lotissements du Comptoir central de crédit dans la commune d'Issy-les-Moulineaux (fin XIX ${ }^{\mathrm{e}}$-début XX $\mathrm{XX}^{\mathrm{e}}$ siècle) », Paris et Ile-de-France, t. XXIX, 1978, pp. 267-295.

Naud, Joseph, Souvenirs de famille, Paris, 1934.

Stoskopf, Nicolas, « Notice n ${ }^{\circ} 12$ : Victor-Corentin Bonnard, 1804-1869 », Banquiers et financiers parisiens, Les patrons du Second Empire, Paris, Picard-Cénomane, 2002.

Vrégille, Gonzague de, « Les banques sans argent », Banque, février 1933, pp. 89-91. 